ARTICLE INFO

Received

March 26, 2021

Revised

June 01, 2021

Accepted

June 03, 2021

Published

July 05, 2021

*Corresponding author Oluwatoba Emmanuel Oyeneyin E-mail

oluwatoba.oyeneyin@aaua.edu.ng emmanueltoba90@gmail.com

Keywords

Nonlinear optical materials

Density functional theory

4-methylphenyl)-(4-

methylpiperidin-1-yl)

methanone

Energy band gap

Molecular hyperpolarizability

How to cite

Oyeneyin OE. Tuning the electronic and nonlinear optical properties of (4methylpheyl) (4methylpiperidin-1-yl) methanone and its substituted analogues. Sci Lett 2021; $9(2): 49-54$
Open Access

\section{Tuning the Electronic and Nonlinear Optical Properties of (4-Methylphenyl) (4-methylpiperidin-1-yl) Methanone and Its Substituted Analogues}

\section{Oluwatoba Emmanuel Oyeneyin*}

Theoretical and Computational Chemistry Unit, Department of Chemical Sciences, Adekunle Ajasin University, Akungba-Akoko, Ondo State, Nigeria

\section{Abstract}

A lot of molecules have been reported for certain applications. However, the need to continuously search for novel materials with more promise is very important because of the competitive technological innovations. One of the ways to achieve this is to use valid theoretical methods that can effectively and accurately predict the properties of existing molecular entities and using these methods to predict the properties of hypothetical molecules. The compound (4-methylphenyl) (4-methylpiperidin-1-yl) methanone (MPMPM) has been reported for its nonlinear optical potentials; however, investigating their reactivity indices would lead to the understanding of the mechanism behind their suitability as nonlinear optical devices. The molecular backbone of MPMPM was altered by introducing some substituents that could alter molecular properties. MPMPM and its derivatives were, therefore, optimized with density functional theory and the time-dependent density functional theory using pure and hybrid correlations and a polar basis set, 6-31G(d). The energy band gaps of the substituted derivatives were lower than that of MPMPM while the dipole moments and hyperpolarizabilities were higher, indicating that they could serve as better alternatives for nonlinear optical applications. 


\section{Introduction}

Organic compounds have been investigated experimentally and theoretically for their use in nonlinear optical (NLO) devices due to the $\pi$-bonds in their moieties, non-centrosymmetric structure and donor-acceptor (D-A) configuration, which all facilitate intra-molecular charge transfer [1-5]. These materials shield human parts (eyes, skin, etc.) and other optical sensors from dangerous laser light by attenuating the incident laser beams [1]. Their use has become very important because of the growing use of intense lasers in the industry, military and medical sectors $[1,2]$. These materials exhibit properties such as two/multi-photon absorption [3, 4], nonlinear scattering [5, 6], reverse saturation absorption $[7,8]$, etc. One of the ways to improve the NLO ability of a known compound is to derivatize it, especially by adding an electron donor at one end and an electron acceptor at the opposite end, this enhances asymmetric charge distribution [9-11]. Asymmetric charge distribution can be investigated by calculating the tensor components via quantum mechanical calculations [12-14]. This is used to calculate the dipole moments and hyperpolarizabilities, which are used to measure their harmonic generation efficiencies. For instance, the molecular first hyperpolarizability is used as a measure of the second harmonic efficiency of a molecule [12]. Density functional theory (DFT) methods have been employed while probing into molecular properties, it has been used in past and in recent times in different areas of materials science research $[11,12,15-21]$. In this study, 4-Methylphenyl) (4-methylpiperidin-1-yl) methanone, (MPMPM; Fig. 1) was synthesized and reported for its NLO ability [22]. This work aimed to corroborate the experimental findings with theoretical method(s) and use the method(s) to predict the NLO properties of derivatized MPMPM to investigate the effect of some substituents on the NLO responses via DFT and TD-DFT calculations. Their NLO responses would be compared to that of urea crystals, a standard for NLO materials.

\section{Materials and Methods}

MPMPM and its derivatives were modeled and optimized using DFT with (i) a restricted KohnSham self-consistent field (SCF) calculation (BLYP) and (ii) a restricted hybrid Hartree FockDFT SCF calculation (B3LYP) [23] using Pulay's direct inversion of the iterative subspace (DIIS) and geometric direct minimization [24] for both correlations and a polar basis set, 6-31G(d) after generating their most stable conformers with the molecular mechanics force field. These levels of theory have been reported to be consistent with experimental trends [25]. The energies of the frontier molecular orbitals (FMOs), that is, energies of the highest occupied molecular orbital, $\mathrm{E}_{\text {номо }}$ and that of the lowest unoccupied molecular orbital, $E_{\text {LUMO }}$ were calculated and used to get the values of the energy band gap, $E_{g}$ (eq. 1), chemical hardness, $\eta$ (eq. 2), chemical softness, $\sigma$ (eq. 3 ) and the dipole moments $(\mu)$ (eq. 4). The molecular first hyperpolarizabilities, $\beta$ were calculated from Kleimann's reduced 10 components from the 27 $3 \times 3 \times 3$ matrix (eq. 5) [26]. The absorption maxima $\left(\lambda_{\text {abs }}\right)$, electronic transition energies/optical band gaps $\left(\mathrm{E}_{\mathrm{ex}}\right)$ and oscillator strengths $(f)$ were calculated using time-dependent density functional theory, TD-DFT method. all on Spartan 14 (v112, Wavefunction, Inc., Irvine.).

$\mathrm{E}_{\mathrm{g}}=\mathrm{E}_{\mathrm{LUMO}}-\mathrm{E}_{\mathrm{HOMO}}$

eq. 1

$\eta=\frac{E_{g}}{2}$

eq. 2

$\sigma=\frac{1}{\eta}$

eq. 3

$\mu=\left(\mu_{x}^{2}+\mu_{y}^{2}+\mu_{y}^{2}\right)^{1 / 2}$ eq. 4

$\beta=\left[\left(\beta_{x x x}+\beta_{x y y}+\beta_{x z z}\right)^{2}+\left(\beta_{y y y}+\beta_{x x y}+\right.\right.$ $\left.\left.\beta_{y z z}\right)^{2}+\left(\beta_{z z z}+\beta_{x x z}+\beta_{y y z}\right)^{2}\right]^{1 / 2} \quad$ eq. 5

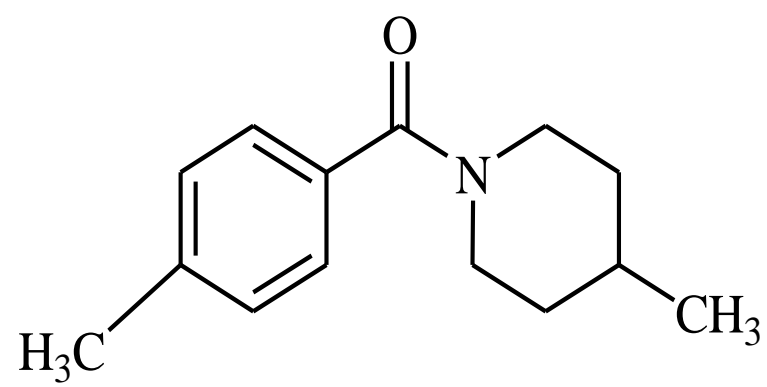

Fig. 14-Methylphenyl) (4-methylpiperidin-1-yl) methanone (MPMPM) and its derivatives (different substituents were used to replace the methyl on phenyl ring). For example, -CHO replaced $-\mathrm{CH}_{3}$ on the phenyl ring to become 4-CHO derivative (Table 1), $\mathrm{CN}$ became 4-CN and so on.

\section{Results and discussion}

\section{Electronic properties}

The electronic properties are presented in Tables 1 and Table 2 for DFT and TD-DFT methods. All substituted derivatives have lower $\mathrm{E}_{\mathrm{g}}$ values than MPMPM with nitro and cyano acrylic acid substituted derivatives having the lowest values, 
Table 1 Electronic and nonlinear optical properties of MPMPM and its substituted derivatives with B3LYP/6-31G*.

\begin{tabular}{cccccccc}
\hline Molecules & Eномо $(\mathbf{e V})$ & $\mathbf{E}_{\mathbf{L u m o}}(\mathbf{e V})$ & $\mathbf{E}_{\mathbf{g}}(\mathbf{e V})$ & $\boldsymbol{\eta}(\mathbf{e V})$ & $\boldsymbol{\sigma}_{\left(\mathbf{e V}^{-1}\right)}$ & $\boldsymbol{\mu}(\boldsymbol{D})$ & $\boldsymbol{\beta}\left(\mathbf{x 1 0} \mathbf{3 0}^{-30} \mathbf{e s u}\right)$ \\
\hline MPMPM & -6.19 & -0.61 & 5.58 & 2.79 & 0.36 & 3.52 & 0.27 \\
4-CHO & -6.55 & -1.99 & 4.56 & 2.28 & 0.44 & 3.80 & 1.08 \\
4-CH=C(CN)COOH & -6.63 & -2.76 & 3.87 & 1.94 & 0.52 & 4.44 & 1.45 \\
4-CN & -6.67 & -1.79 & 4.88 & 2.44 & 0.41 & 5.73 & 2.64 \\
4-NO & -6.73 & -2.58 & 4.15 & 2.08 & 0.48 & 5.87 & 1.44 \\
4-NC & -6.58 & -1.55 & 5.03 & 2.52 & 0.39 & 5.06 & 2.39 \\
\hline
\end{tabular}

Table 2 Electronic and nonlinear optical properties of MPMPM and its substituted derivatives with BLYP/6-31G*.

\begin{tabular}{cccccccc}
\hline Molecules & $\mathbf{E}_{\text {номо }}(\mathbf{e V})$ & $\mathbf{E}_{\mathbf{L u m o}}(\mathbf{e V})$ & $\Delta \mathbf{E}(\mathbf{e V})$ & $\boldsymbol{\eta}(\mathbf{e V})$ & $\boldsymbol{\delta}\left(\mathbf{e V}^{-\mathbf{1}}\right)$ & $\boldsymbol{\mu}(\boldsymbol{D})$ & $\boldsymbol{\beta}\left(\mathbf{x 1 0} \mathbf{3}^{-30} \mathbf{e s u}\right)$ \\
\hline MPMPM & -4.82 & -1.15 & 3.67 & 1.84 & 0.55 & 3.43 & 0.25 \\
4-CHO & -5.21 & -2.50 & 2.71 & 1.36 & 0.74 & 4.05 & 1.14 \\
4-CH=C(CN)COOH & -5.31 & -3.15 & 2.16 & 1.08 & 0.93 & 4.69 & 1.43 \\
4-CN & -5.32 & -2.26 & 3.06 & 1.53 & 0.65 & 5.83 & 2.65 \\
4-NO & -5.39 & -3.09 & 2.30 & 1.15 & 0.87 & 5.99 & 1.43 \\
4-NC & -5.23 & -2.06 & 3.17 & 1.59 & 0.63 & 5.24 & 2.50 \\
\hline
\end{tabular}

which could be attributed to the larger molecular weight of the substituted derivatives, mesomeric effects and/or conjugation length. The substituted derivatives are, therefore, more reactive than MPMPM. Nitro is a stronger electron-withdrawing group than the others (increase mesomeric effect) while cyano acrylic acid has the longest $\pi$ conjugation length and even the largest of all the molecules. The $\eta$ and $\sigma$ values are expected to have similar and reverse trends, respectively (eq. 2 and eq. 3). Consequently, all substituted derivatives have lower $\eta$ values, with nitro and cyano acrylic acid having the lowest, while the highest $\sigma$ values. Formation of instantaneous dipoles (tensor components) is usually enhanced by improved reactivity of molecules (the closer the HOMOLUMO gap) and the $\mu$ values of the substituted molecules are higher than that of MPMPM (Tables 1 and 2) and all molecules under investigation. MPMPM showed higher $\mu$ values than urea $(1.98 \mathrm{D})$ [27].

\section{Hyperpolarizability}

The $\beta$ value is a measure of the second harmonic generation (SHG) efficiency of a NLO molecule. These values are higher in the substituted derivatives than MPMPM. A similar trend was observed in the two correlations used. The $\beta$ value of urea (a standard for organic NLO materials) was calculated as $0.6498 \times 10^{-30}$ esu [12]. From Table 1 and Table 2, all substituted derivatives have higher values than urea, this is because of the enhanced delocalization of charges brought about by the different substituents that reduced the HOMO-

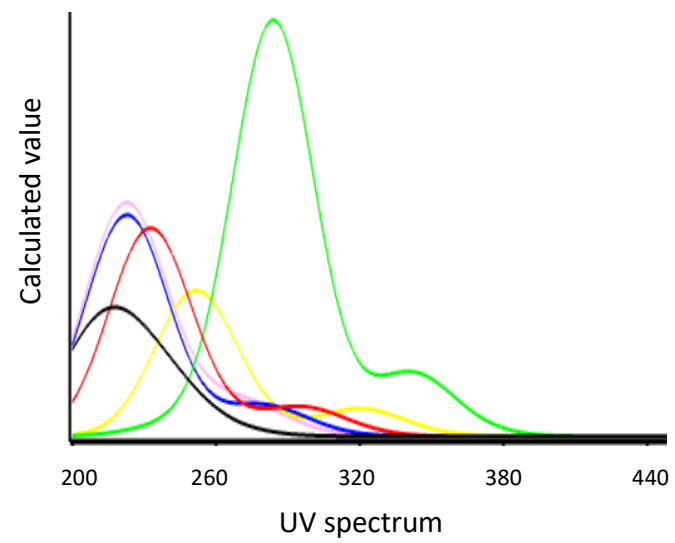

Fig. 2 Calculated UV spectra of the MPMPM and its substituted derivatives $(\mathrm{MPMPM}=$ black; $4-\mathrm{CHO}=$ red; $4-$ $\mathrm{CH}=\mathrm{C}(\mathrm{CN}) \mathrm{COOH}=$ green; $4-\mathrm{CN}=$ blue; $4-\mathrm{NO}_{2}=$ yellow; $4-$ $\mathrm{NC}=$ pink) with hybrid correlation.

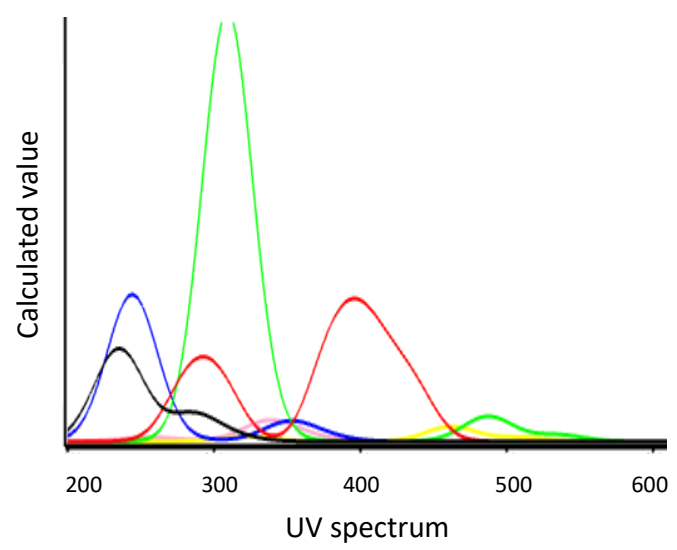

Fig. 3 Calculated UV spectra of the MPMPM and its substituted derivatives $($ MPMPM = black; 4-CHO = red; $4-$ $\mathrm{CH}=\mathrm{C}(\mathrm{CN}) \mathrm{COOH}=$ green; $4-\mathrm{CN}=$ blue; $4-\mathrm{NO}_{2}=$ yellow; $4-$ $\mathrm{NC}=$ pink) with pure correlation. 

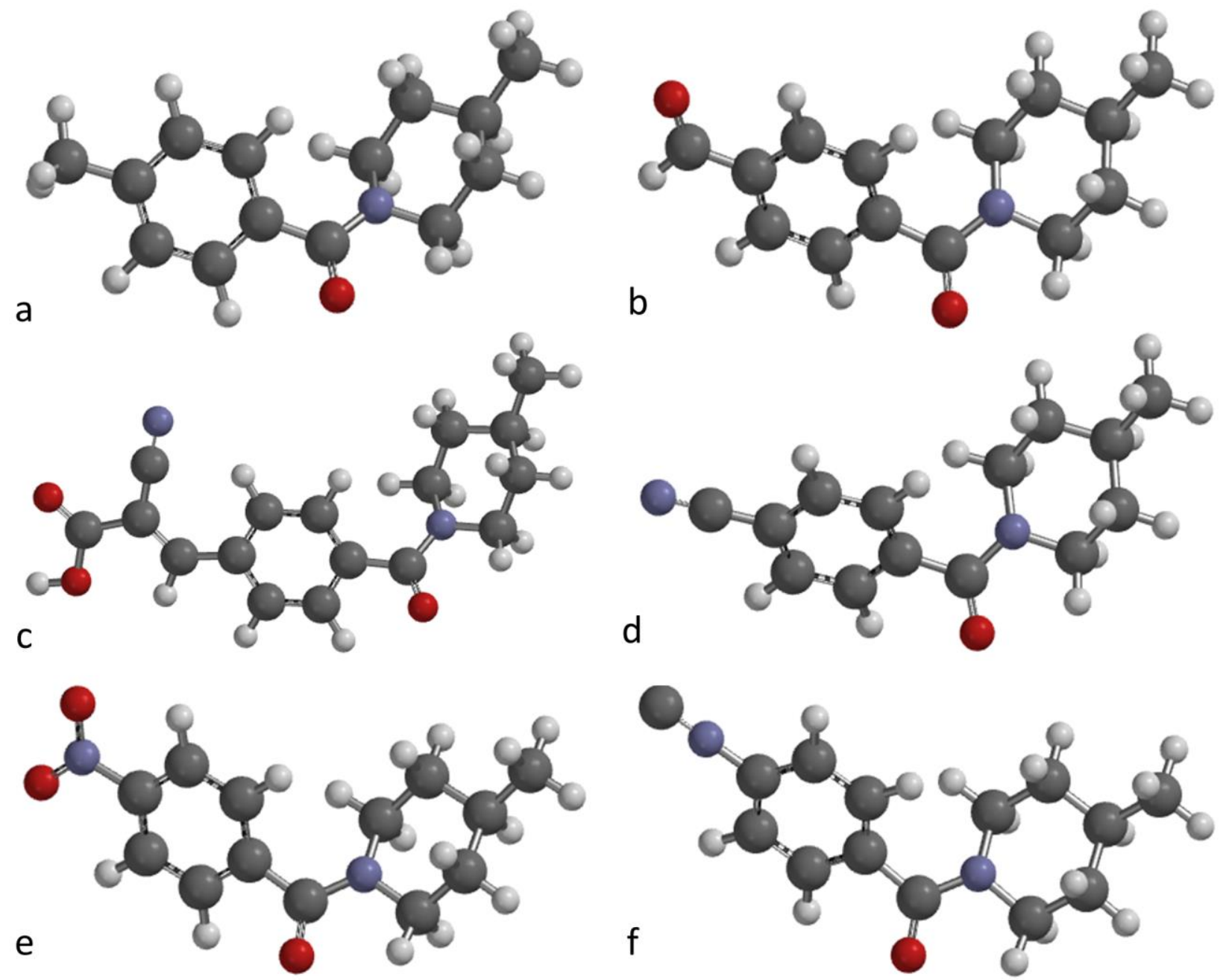

Fig. 4 Optimized (4-methylphenyl) (4-methylpiperidin-1-yl) methanone (MPMPM) (a); optimized cyano derivative (b); optimized 4-CHO derivative (c); optimized nitro derivative (d); optimized 4-cyano acrylic acid (e); and optimized isocyano derivative (f).

LUMO gap and improved intramolecular charge transfer (ICT). The chemical hardness and softness values provide information about the molecules' reactivity and electron transportation. All the substituted derivatives are softer than MPMPM as a result of the lower energy band gap. This consequently led to an increase in their dipole moment values and hyperpolarizabilities.

\section{Electronic transitions}

MPMPM was absorbed at $285 \mathrm{~nm}$ with BLYP correlation, while it was absorbed at $237 \mathrm{~nm}$ with B3LYP (Fig. 2 \& Fig. 3). The absorption of $295 \mathrm{~nm}$ was ignored because of its zero-oscillator strength. The experimental $\lambda_{\text {abs }}$ was $296 \mathrm{~nm}$ [22]. The band gap corresponding to the calculated $\lambda_{\text {abs }}$ (BLYP) was $4.34 \mathrm{eV}$ while the experimental band gap obtained was $4.19 \mathrm{eV}$. The calculated $\lambda_{\text {abs }}$ (BLYP) corresponding to $295 \mathrm{~nm}(4.21 \mathrm{eV})$ was ignored because of zero oscillator strength. The substituted derivatives bathochromically shifted the $\lambda_{\text {abs }}$ (Fig. 4, $\&$ Tables S1-S6). Cyano acrylic acid substituted derivative shifted the $\lambda_{\text {abs }}$ the most, followed by $\mathrm{NO}_{2}$. Similar results were observed in previous studies [28]. The red shift observed in 4$\mathrm{CH}=\mathrm{C}(\mathrm{CN}) \mathrm{COOH}$ is attributed to its conjugation length and also the strong inductive electronaccepting nature of nitro derivative than others [29, 30].

\section{Conclusions}

DFT and TD-DFT calculations were carried out on MPMPM and its substituted derivatives to obtain the ground state optimized geometry, FMOs energies, energy band gap, dipole moments and hyperpolarizabilities. The properties calculated 
were used to explain their NLO properties and compared with the values of urea, a standard NLO material. The FMOs energies obtained show that the substituted derivatives are more reactive than MPMPM owing to lower energy band gaps. The dipole moments of the molecules were higher than that of urea while the hyperpolarizability values of the substituted derivatives were higher than that of urea in multiple folds. The hyperpolarizability of MPMPM and derivatives depends on the electronic structure of the molecule, as seen from the lower energy band gap, which increased charge transfer from the donor to the acceptor parts of the molecules. It is also evident that the hyperpolarizability was affected by the energy band gap. The substituted derivatives could be used as precursors in the development of better NLO materials. In this study, only the electronic components of the hyperpolarizability values were considered. The effect of field strength on nuclear positions was not considered.

\section{Conflict of Interest}

There are no conflicts of interest to declare.

\section{Acknowledgment}

We pay gratitude to Adekunle Ajasin University, Akungba-Akoko, Ondo State for creating an enabling environment for this research work.

\section{References}

[1] Veisi M, Kazemi SH, Mahmoudi M. Tunnelinginduced optical limiting in quantum dot molecules. Sci Rep 2020; 10:1-10.

[2] Nair SS, Thomas J, Suchand Sandeep CS, Anantharaman MR, Philip R. An optical limiter based on ferrofluids. Appl Phys Lett 2008; 92:171908-2.

[3] Johnsen M, Ogilby PR. Effect of solvent on twophoton absorption by vinyl benzene derivatives. J Phys Chem A 2008; 112:7831-7839.

[4] Drobizhev M, Makarov NS, Stepanenko Y, Rebane A. Near-infrared two-photon absorption in phthalocyanines: Enhancement of lowest geradegerade transition by symmetrical electron-accepting substitution. J Chemical Phys 2006; 124:1-12.

[5] Skumanich A, Jurich M, Swalen JD. Absorption and scattering in nonlinear optical polymeric systems. Appl Phys Lett 1993; 62:446-448.

[6] Joulaud C, Mugnier Y, Djanta G, Dubled M, Marty JC, Galez C, et al. Characterization of the nonlinear optical properties of nanocrystals by hyper rayleigh scattering. J Nanobio 2013; 11:1-9.

[7] Xia T, Hagan DJ, Dogariu A, Said AA, Van Stryland EW. Optimization of optical limiting devices based on excited-state absorption. Appl Opt 1997; 36:4110.
[8] Yadav C, Roy S. Optimization of ultrafast reverse saturable to saturable absorption transition in $\mathrm{Ru}$ dioxolene complex for all-optical logic applications. Opt Quant Electron 2017; 49:1-11.

[9] Oyeneyin OE, Adejoro IA, Ogunyemi BT, Esan OT. Structural and solvent dependence on the molecular and nonlinear optical properties of 10-octyl thiophene-based phenothiazine and substituted derivatives - a theoretical approach. J Taibah Univ Sci 2018; 12:483-493.

[10] Adejoro IA, Oyeneyin OE, Ogunyemi BT. Computational investigation on substituent and solvent effects on the electronic, geometric and spectroscopic properties of azobenzene and some substituted derivatives. Int $\mathbf{J}$ Comput Theor Chem 2015; 3:50-57.

[11] Islam $\mathrm{N}$, Lone IH. Computational studies on optoelectronic and nonlinear properties of octaphyrin derivatives. Front Chem 2017; 5:1-11.

[12] Adhikari S, Kar T. Experimental and theoretical studies on physicochemical properties of L-leucine nitrate-a probable nonlinear optical material. J Crys Grow 2012; 356:4-9.

[13] Afzal SM, Razvi MAN, Khan SA, Osman OI, Bakry AH, Asiri AM. Physicochemical and nonlinear optical properties of novel environmentally benign heterocyclic azomethine dyes: experimental and theoretical studies. PLoS One 2016; 11:1-25.

[14] Zhang L, Qi, D, Zhao L, Chen C, Bian Y, Li W. Density functional theory study on subtriazaporphyrin derivatives: Dipolar/octupolar contribution to the second-order nonlinear optical activity. J Phys Chem A 2016; 116:10249-10256.

[15] Oyeneyin OE, adejoro IA, Esan TO. Substituent Effects on the structural and nonlinear optical properties of 1-[4-(\{(E)-[4-(methylsulfanyl)phenyl] methylidene \}amino)phenyl] ethanone and some of its substituted derivatives- a theoretical method. Phys Chem Res 2018; 6:667-683.

[16] Semire B, Oyebamiji AK, Odunola OA. Electronic properties' modulation of $\mathrm{D}-\mathrm{A}-\mathrm{A}$ via fluorination of 2-cyano-2-pyran-4-ylidene-acetic acid acceptor unit for efficient DSSCs: DFT-TDDFT approach. Scient Afric 2020; 7:e00287.

[17] Oyebamiji AK, Oyedeji FO, Adejoro IA, Adeleke BB. Computational studies of some hydrazone derivatives as antibacterial agent: $\mathrm{dft}$ and docking methods. J Pure Appl Chem Res 2019; 8:1-6.

[18] El-shamy AM, El-hadek MA, Nassef AE, El-bindary RA. Box-Behnken design to enhance the corrosion resistance of high strength steel alloy in $3.5 \mathrm{wt} . \%$ $\mathrm{NaCl}$ solution. Morocc J Chem 2020; 8:788-800.

[19] El-shamy AM, El-hadek MA, Nassef AE, El-bindary RA. Optimization of the influencing variables on the corrosion property of steel alloy 4130 in $3.5 \mathrm{wt} . \%$ nacl solution. J Chem. 2020: 1-20.

[20] Onawole AT, Nasser MS, Hussein IA. Theoretical studies of methane adsorption on silica-kaolinite interface for shale reservoir application applied surface science theoretical studies of methane adsorption on silica-kaolinite interface for shale reservoir application. Appl Surf Sci 2012; 
546:149164.

[21] Yusuf TL, Oladipo SD, Olagboye SA, Zamisa SJ, Tolufashe GF. Solvent-free synthesis of nitrobenzyl Schiff bases: Characterization, antibacterial studies, density functional theory and molecular docking studies. J Mol Struc 2020; 1222:128857.

[22] Priya MK, Revathi BK, Renuka V, Asirvatham PS. Synthesis, structure elucidation, spectroscopic analysis, thermal and NLO properties of A new piperidine derivative-(4-methylphenyl) (4methylpiperidin-1-yl) methanone. Opt Las Tech 2019; 111:616-622.

[23] Becke AD. Density-functional thermochemistry. III. The role of exact exchange. J Chem Phys 1993; 98:5648-5652.

[24] Pulay P. Convergence acceleration of iterative sequences. the case of scf iteration. Chem Phys Lett 1980; 73:393-398.

[25] Amoko SJ, Akinyele OF, Oyeneyin OE, Olayanju DS, Aboluwoye CO. Experimental and theoretical investigation of corrosion inhibitive potentials of $(\mathrm{E})$ 4-hydroxy-3-[(2,4,6-tribromophenyl)diazenyl] benzaldehyde on mild steel in acidic media. Phys
Chem Res 2020; 8:399-416.

[26] Kleinmann DA. Nonlinear dielectric polarization in optical media. Phys Rev 1962; 2:1977-1979.

[27] Shanmugam G, Thirupugalmani K, Kannan V, Brahadeeswaran S. Spectroscopic, quantum-chemical and $\mathrm{x}$-ray diffraction studies of piperidinium $\mathrm{p}$ hydroxybenzoate-combined experimental and theoretical studies on a novel NLO crystal. Spectro Acta- Part A: Mol Biomol Spec 2013; 106:175-184.

[28] Oyeneyin OE, Adejoro IA, Obadawo BS, Amoko JS, Kayode IO, Akintemi EO, et al. Investigation into the molecular properties of 3-(4-hydroxyphenyl)prop-2en-1-one 4-phenyl schiff base and some of its derivatives-DFT and molecular docking studies. Sci Lett 2020; 9:4-11.

[29] Appleton AL, Brombosz SM, Barlow S, Sears JS, Bredas JL, Marder SR, et al. Effects of electronegative substitution on the optical and electronic properties of acenes and diazaacenes. Nat Comm 2010; 1:91.

[30] Jezuita A, Ejsmont K. Substituent effects of nitro group in cyclic compounds. Struc Chem 2021; 32:179-203. 